\title{
Study on the Nonverbal Behavior in Psychological Counseling
}

\author{
Lu Yang
}

Liaoning Jianzhu Vocational College, Liaoyang, Liaoning, 111099

Keywords: nonverbal behavior; psychological counseling; research effect

\begin{abstract}
In the process of psychological counseling, verbal communication is the most common way. However, when there are obstacles in speech communication, the important information of visitors may unconsciously manifest themselves through a series of nonverbal actions such as micro-expressions and body movements. Therefore, it is very important for consultants to master relevant knowledge of non-verbal behavior and apply them in the process of psychological counseling. The study found that the analysis of facial expressions, body movements, and sound characteristics in non-verbal behaviors not only facilitates the smooth progress of the consultation process, but also helps the consultants find the visitors' implicit information. This paper analyzes the application of non-verbal behavior in psychological counseling and explores its research value. It aims to help consultants to better conduct psychological counseling work so as to achieve the best counseling effect.
\end{abstract}

\section{Introduction}

With the development of the times, more and more psychologists have realized the importance of nonverbal behavior in psychological counseling. The nature of psychological counseling is actually a kind of person-to-person communication. This kind of communication is mainly verbal communication and non-verbal communication. Verbal communication is the direct expression of personal information and ideas through language symbols. It is the most important way for the two parties to exchange information. Non-verbal communication communicates ideas and expresses emotions through a series of non-verbal actions such as: eyes, facial expressions, body postures and movements, and changes in voice and intonation. However, when many complex emotions and rich psychological activities are difficult to express through verbal communication, people's implicit information tends to be expressed unconsciously through nonverbal behavior. Therefore, when consultants and consultants have communication problems during the consultation process, or when consultants want to learn more about the consultants, using non-verbal information will help them find potential problems. If consultants can perceive their hidden meanings in the subtle non-verbal behaviors of the visitors, and then penetrate into his inner world, they will be helpful for consulting work. Therefore, the application of non-verbal information to psychological counseling has a wide range of uses [1].

\section{Definition and Classification of Non-verbal Behavior}

Compared with verbal behavior, nonverbal behaviors convey some unique properties: (1) Uncontrollability. Non-verbal behavior will reveal the true emotional state, intentions and personality characteristics of the person in the unconscious, and because the individual can not see their non-verbal behavior in general, the individual non-verbal behavior is not easily trained and changed. (2) Easy to express. Sometimes words are not easy to express and non-verbal acts are more easily expressed. For example, frowning is much easier than criticizing the other party or expressing "disagree" directly; and nonverbal behavior can increase the continuity of conversation. Sexuality, for example, when two people talk, the speaker's common eyes and intonations indicate who the following speech is (Language conversion, as in linguistics [2]; (3) Consistency across cultures. Studies have shown that the same emotions are expressed in different races. Emotions are judged by emotions in groups of the same cultural background more than in different cultural 
backgrounds. Accurate; (4) Cross-cultural differences. Culture will shape the way people express their emotions, which leads to nuances in the expression of emotional non-verbal behaviors due to cultural differences; in addition, the number of individual non-verbal behaviors varies from culture to culture, and Kim Liang and Li analyzed the videos of consultations of five Asian-American female consultants and five European-American female consultants and found the frequency of adaptors, posture transitions, and smiles of European-American consultants. Obviously more than Asian American consultants. Because of the uniqueness of non-verbal communication in conveying information, its important role in psychological counseling is mainly reflected in the following six aspects: (1) Repeat, correct, supplement or regulate one's speech acts), counselors can express unconditional positive concerns better with warm, open and non-verbal behavior combined with verbal behavior; (2) control of talks. Talks are initiated through interpersonal distances, expressions, and eye contact; talks are maintained through the use of gestures, eye contact, and tones; and the talks are ended by diverting sight and the like. In the consultation, the counselor not only expresses his or her interest in the talks through eye contact. Can better understand the subject's feelings about the subject of the conversation through the eyes of the parties; (3) Affect the atmosphere of the meeting. Deep breathing during the talks, as well as restraining breathing, will affect the atmosphere of the talks [3]. In counseling, the counselor can adjust the atmosphere of the meeting through non-verbal actions such as gestures and other body movements, and perceive the parties' feelings about the meeting through non-verbal actions of the parties. (4) Express emotions. Express your emotions through facial expressions, intonation, etc.; (5) Maintain human relationships. You can express friendly, nervous or relaxed consulting relationships through non-verbal behavior, and develop trust through eye contact to promote the development of intimate counseling relationships; (6) Shaping the individual image of consultants.

\section{The Use of Non-verbal Behavior in Psychological Counseling}

Mankind has a rich expression, and expression is another sensor of the human heart's emotional expression. Under certain circumstances, people's emotions can be directly expressed without any speech information. Therefore, it is possible for people to perceive emotions and anticipate behaviors by virtue of their facial expressions. Therefore, in psychological counseling, the counselor may be able to detect the effectiveness of his/her consultation by changing the face of the interviewer, so that he can adjust the content, steps, and methods of his consultation at any time. For example, in the consultation process, the counselor can help to verify whether the visitor's inference and guess are accepted by observing the visitor's non-verbal behavior: if the visitor shows a faint smile to the counselor, but half Closed or low-lying eyelids, eyebrows gently curved into an arc, indicating that visitors have not completely opened their hearts to the consultants. In short, when faced with visitors, the counselor must pay attention to his facial expressions, consider the changes in the various parts of the eyes, ears, nose and nose, and the relationship with his psychological reactions. Only when they are fully integrated can they be examined. Determine the actual psychological response of the visitors [4].

All kinds of movements and behaviors of human beings are caused by the central nervous system of the brain to reach various regions. Therefore, human body postures will inadvertently reveal the actual psychological state of the visitors. For example, if a visitor overlaps his arms, crosses his legs, leans his head forward, his body moves slightly to sideways, and his tongue and hands are thrown, it almost means that the visitor refuses advice and help from the counselor. Therefore, the consultant must pay attention to the various actions of the visitors during the consultation process. These details will help the counselors to further step into the inner world of the visitors. Because of the difference in tone and tone, each person has a strong personal attribute when he speaks, and uses his rhythm and speed to help express his feelings and release his inner feelings. Therefore, in the process of psychological counseling, the counselor can understand the information of the visitors through the features of the voice. For example, when a visitor speaks a loud voice and has a high pitch and a fast rhythm, this may express his annoyance and anxiety; and when his voice becomes weaker and weaker, it may indicate that he is not happy or disappointed at this time. However, due 
to the different geographical and environmental conditions, the accent and speech habits of visitors may affect the judgment of the counselor. Therefore, if the counselor wants to help the two parties communicate and promote communication through the non-verbal behavior of the visitors, detailed and comprehensive observation and analysis of the various behaviors and information of the visitors are required. The judgement cannot be judged only by experience and subjective impression. , otherwise it will make the consultation go passive or even fail.

Since the education background, living environment, gender differences, language habits, and religious beliefs of the consultant and the visitor are different, the language level of the two parties may differ. Even the same context can lead to changes in feelings and emotions on both sides, and even communication problems and unnecessary conflicts. At this point, the application of non-verbal behavior will help to break the barriers to communication and reduce unnecessary conflicts. Sometimes the amount of information reflected by non-verbal behavior is far better than words, and it not only makes verbal information more vivid through various changes in facial expressions, body movements, and sounds, but also facilitates communication between patients and patients [5].

Human expression information is not only through speech, but also uses various movements, expressions, and moods of the body to help enhance the persuasiveness of expression. These nonverbal actions may be intentional or unintentional. At the same time, speech is also masked. Visitors may say something that is contrary to their innermost feelings, but they show through nonverbal behavior. Therefore, if the counselor wants to make his counseling pertinent and effective, he must not only listen carefully to the visitor's speech information, but must also be good at capturing his subtle non-verbal information and gain insight into the hidden meaning of the interviewer, thereby deepening the visitors' The inner world so that the consultation can be carried out. The ancients said that "words are the heartfelt thoughts". In terms of psychotherapy, what the client is talking about is the expression of his inner state. There is no doubt about this. However, this "heartfelt" has the difference of modification or not. One person may hate his job, but for some reason, he can only say that he likes the job. This is the filtration and modification of the inner defense. It is not the true feeling of the heart, and the original Annoying can only be suppressed in my heart. Under normal circumstances, this will not be a big problem, but if it is suppressed so much that psychological disorders occur, then in treatment, expressing this long-standing apathetic emotion that has been deposited in the heart, that is, catharsis, becomes An important content. Anna $\mathrm{O}$, a famous patient in the history of psychoanalysis, had symptoms of inability to drink water during the course of his illness. After treatment, the symptoms disappeared. From this we can see the effect of this catharsis. To achieve the effect of catharsis, first of all, the therapist needs to be able to accurately understand the emotional state of the client's heart and help them clarify the meaning of the state and the impact on their behavior. In this regard, the non-verbal expression of the client often provides valuable information to the therapist. The so-called "human body hardly knows how to harmonizely lie". For example, a quiet little girl at home is very obedient at home, but always has problems when the mother tells her to do something: either she forgets quickly, or she breaks things like cups and bowls. This kind of behavior is not carelessness. It is an expression of the hostility of the little girl to the mother. This kind of hostility is suppressed in the family. When the therapist understood the meaning of these behaviors, when the treatment prompted the little girl to more fully express this negative emotion, her communication with her mother gradually improved. In psychotherapy, nonverbal behaviors include expressions, gaze, tone of speech, hesitation in narration, and interruptions, gestures, and body postures. From these aspects we can learn a lot of information from the client, such as whether the client is nervous or relaxed? Is it offensive or defensive or evasive?

\section{Conclusion}

The application of nonverbal behavior to psychological counseling not only helps counselors better understand the actual feelings of visitors, but also facilitates the exchange of information between the two parties and establishes a good relationship between them. Therefore, non-verbal 
behavior has a strong use value, but it must be considered in many aspects when it is used. It must not be used blindly.

\section{References}

[1] McGrath, J. M., Arar, N. H., \& Pugh, J. A. Theinfluence of electronic medical record usage on nonverbalcommunication in the medical interview [J]. HealthInformatics Journal, 13(2), 105-118.

[2] Murphy, N. A., Hall, J. A., Mast, M. S., Ruben, M. A., Frauendorfer, D., Blanch-Hartigan, D., Nguyen, L. Reliability and validity of nonverbal thin slices insocial interactions [J]. Personality and Social PsychologyBulletin, 41(2), 199-213.

[3] Navarretta, C. Feedback facial expressions andemotions [J]. Journal on Multimodal User Interfaces, 8(2),135-141.

[4] Osmun, W. E., Brown, J. B., Stewart, M., \& Graham, S. (2000). Patients' attitudes to comforting touch in familypractice [J]. Canadian Family Physician, 46, 2411-2416.

[5] Pawlikowska, T., Zhang, W. J., Griffiths, F., van Dalen, J., \&van der Vleuten, C. Verbal and non-verbalbehavior of doctors and patients in primary careconsultations-How this relates to patient enablement [J]. Patient Education and Counseling, 86(1), 70-76. 